\title{
Some remarks on the free fields realization of the bosonic string on $A d S_{3}$.
}

\author{
Igor Pesando * \\ Dipartimento di Fisica Teorica, Universitá di Torino \\ Istituto Nazionale di Fisica Nucleare (INFN) - sezione di Torino, Italy \\ via P. Giuria 1, I-10125 Torino \\ ipesando@to.infn.it
}

Abstract: We discuss the classical solutions of the equations of motion and the possible boundary condition for a bosonic string with Kalb-Ramond background in $A d S_{3}$. It turns out that there exists three different physical sectors and that it is also possible to describe the motion on an extremal black hole background. The existence of three sectors clearly shows how one of the spectra proposed is incomplete. We consider also the classical 'canonical' transformation which maps the string fields to the classical Wakimoto ones. It turns however out that the Wakimoto fields are not free because of the boundary conditions and in order to have the usual mode expansion with reasonable behaviour under complex conjugation it is necessary to consider the complexification of $A d S_{3}$ and then add some constraints. Furthermore they cover only half $A d S_{3}$ and we need different patches to cover the whole space and to make the above mentioned transformation really canonical.

\section{Introduction.}

The last year has seen an increasing and renewed interest in string theory propagating on $A d S$ backgrounds. A particular attention has been dedicated to the $A d S_{3}$ background with NSNS flux, this because it is the only non trivial model which has been possible to treat exactly at the quantum level. Nevertheless and despite its apparent simplicity our understanding is far from being complete. In particular the question of which is its spectrum is not satisfactorily settled. It was already noted in the old days that with a naive quantization the Virasoro constraints were not sufficient to eliminate the ghost from the spectrum $([\overline{1}|\overline{1}|)$. In order to remedy to this unfortunate circumstance two different proposals have been put forward ${ }^{1}$

\footnotetext{
*Preprint DFTT 09/00; Work supported partially by the European Commission TMR programme ERBFMRXCT96-0045.

${ }^{1}$ Some recent papers have done some steps forwards in increasing our understanding of the quantum theory

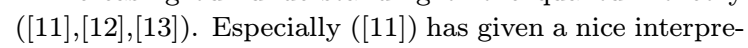

1. To truncate the spectrum to the unitary part: this was the approach first advocated by Petropolous (

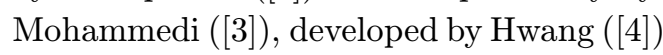
and collaborators. Last year Evans, Gab-

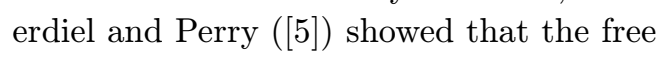
spectrum is actually ghost free;

2. To introduce some new hidden dof in form of zero momenta of the fields used to bosonize the KM currents. This way was first undertaken by Bars $\left(\left[\overline{6}_{0}^{0}\right]\right)$ and developed by Satoh

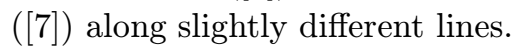

We think that both proposals have some weak sides but the main criticism is due to their philosophical attitude to the problem (see also ( for a recent review of the open problems): string theory is so consistent that there should not be necessary to introduce new elements in the game since the string itsself should give the answer.

tation of the old results by $\left(\left[\bar{u}_{i}\right]\right)$ on the construction of a modular invariant partition function. 
Our approach is different from both the previous ones even if it is closer to the second one: we find the classical canonical transformation from the string fields to the Wakimoto fields and then we discuss how and in what extent the Wakimoto fields are free.

\section{The Classical Bosonic String The- ory on $\mathrm{AdS}_{3}$.}

We first exam the equations of motion and boundary conditions for a classical bosonic string with a Kalb-Ramond background thought of as a WZW theory plus Virasoro constraints not relying on the KM symmetry structure present in the theory and we derive the general solution. We set up the canonical formalism which we use to discuss the classical 'canonical' transformation to the classical Wakimoto fields. finally we discuss the boundary conditions and their consequences. It turns out that the Wakimoto fields while satisfying free fields equations of motion are generically not free because of the boundary conditions; we discuss how to circumvent the problem.

\subsection{The action and the constraints of the bosonic string on $A d S_{3}$.}

The string action is given $b^{2}$

$$
\begin{aligned}
S_{w z w}= & \frac{|k|}{4 \pi} \int d^{2} \xi \operatorname{tr}\left(\omega_{+} \omega_{-}\right) \\
& +\frac{k}{12 \pi} \int \operatorname{tr}\left(\omega^{3}\right)
\end{aligned}
$$

where $\omega=g^{-1} d g=\omega_{+} d \xi^{+}+\omega_{-} d \xi^{-}$is the pullback on the string worldsheet of the left invariant

- Spacetime coordinates: $x^{ \pm}=\frac{x^{1} \pm x^{0}}{\sqrt{2}}$

- WS metric $-\eta_{00}=\eta_{11}=1 ; \eta_{+-}=\frac{1}{2} \eta^{+-}=2$

- WS coordinates: $\xi^{ \pm}=\tau \pm \sigma ; \sigma \in[0,2 \pi] ; d^{2} \xi=$ $2 d \tau d \sigma ; \partial_{ \pm}=\frac{1}{2}\left(\partial_{\tau} \pm \partial_{\sigma}\right)$

- Sigma matrices: $\sigma_{3}=\left(\begin{array}{cc}1 & \\ & -1\end{array}\right) \sigma_{+}=$ $\left(\begin{array}{l}1 \\ 0\end{array}\right) \sigma_{-}=\left(\begin{array}{ll} & 0 \\ 1 & \end{array}\right)$

- $\operatorname{sl}(2, R)$ algebra: $\sigma_{a} \sigma_{b}=\epsilon_{a b c} \sigma^{c}+\eta_{a b} 1$ with $\epsilon_{+-3}=\frac{1}{2}$ and $\eta_{+-}=\frac{1}{2} \eta_{33}=1$ one form on the group $S L(2, R)$. When we use the explicit expression for $g$ in the Gauss form ${ }^{3}$

$g=\left(\begin{array}{cc}1 & \\ x^{-} & 1\end{array}\right)\left(\begin{array}{cc}e^{\rho} & \\ & e^{-\rho}\end{array}\right)\left(\begin{array}{cc}1 & x^{+} \\ & 1\end{array}\right)=\left(\begin{array}{cc}e^{\rho} & e^{\rho} x^{+} \\ e^{\rho} x^{-} & e^{-\rho}+e^{\rho} x^{+} x^{-}\end{array}\right)$

and take $k=-|k|$ the previous expression for the action becomes

$$
S_{w z w}=\frac{|k|}{2 \pi} \int d^{2} \xi \partial_{+} \rho \partial_{-} \rho+e^{2 \rho} \partial_{-} x^{+} \partial_{+} x^{-}
$$

We can interpret this as a string action in the conformal gauge if we add the Virasoro constraints

$$
\begin{aligned}
& T_{++}=|k|\left[\left(\partial_{+} \rho\right)^{2}+e^{2 \rho} \partial_{+} x^{+} \partial_{+} x^{-}\right]=0 \\
& T_{--}=|k|\left[\left(\partial_{-} \rho\right)^{2}+e^{2 \rho} \partial_{-} x^{+} \partial_{-} x^{-}\right]=0
\end{aligned}
$$

We want now to derive the the equations of motion and the allowed boundary conditions associated with the action (2.3i) in the same way of we proceed with the usual string action in Minkowski space. The equations of motion read

$$
\begin{array}{r}
\partial_{-}\left(e^{2 \rho} \partial_{+} x^{-}\right)=\partial_{+}\left(e^{2 \rho} \partial_{-} x^{+}\right)=0 \\
\partial_{-} \partial_{+} \rho+e^{2 \rho} \partial_{-} x^{+} \partial_{+} x^{-}=0
\end{array}
$$

while from the request of the cancellation of the surface terms obtained from the fields variation we get the boundary conditions

$$
\begin{aligned}
\left.\delta \rho\right|_{\sigma=0}=\left.\delta \rho\right|_{\sigma=2 \pi} & \Rightarrow \\
\left.\rho^{\prime}\right|_{\sigma=0} & =\left.\rho^{\prime}\right|_{\sigma=2 \pi} \\
\left.\delta x^{-}\right|_{\sigma=0}=\left.\delta x^{-}\right|_{\sigma=2 \pi} & \Rightarrow \\
\left.e^{2 \rho} \partial_{-} x^{+}\right|_{\sigma=0} & =\left.e^{2 \rho} \partial_{-} x^{+}\right|_{\sigma=2 \pi}(2 . \\
\left.\delta x^{+}\right|_{\sigma=0}=\left.\delta x^{+}\right|_{\sigma=2 \pi} & \Rightarrow \\
\left.e^{2 \rho} \partial_{+} x^{-}\right|_{\sigma=0} & =\left.e^{2 \rho} \partial_{+} x^{-}\right|_{\sigma=2 \pi}
\end{aligned}
$$

Anticipating the expressions for the KM currents $\left(2 . \overline{1}+2 . \overline{1} \overline{1}_{1}^{\prime}\right)$ we can rewrite the last two conditions $(2.6,2.71)$ as

$$
\left.J^{-}\right|_{\sigma=0}=\left.\left.J^{-}\right|_{\sigma=2 \pi} \quad \bar{J}^{+}\right|_{\sigma=0}=\left.\bar{J}^{+}\right|_{\sigma=2 \pi}
$$

${ }^{3}$ This expression only covers half $A d S_{3}$, even if we perform an analytic continuation letting $e^{\rho} \in R$ they do not cover the whole manifold since points like $\left(\begin{array}{cc}\alpha & \beta \\ -\frac{1}{\beta} & 0\end{array}\right)$ are left out. 


\subsection{The general solution of the equations of motions.}

We are now ready to discuss the general solution of eq.s $(2.4-2.51)$. We can write the general solution as

$$
\begin{aligned}
x^{+}= & a\left(\xi^{+}\right)+e^{-2 c\left(\xi^{+}\right)} \frac{\bar{b}\left(\xi^{-}\right)}{1+\bar{b}\left(\xi^{-}\right) b\left(\xi^{+}\right)}(2.8) \\
x^{-}= & \bar{a}\left(\xi^{-}\right)+e^{-2 \bar{c}\left(\xi^{-}\right)} \frac{b\left(\xi^{+}\right)}{1+\bar{b}\left(\xi^{-}\right) b\left(\xi^{+}\right)} \\
\rho= & \lg \left(1+\bar{b}\left(\xi^{-}\right) b\left(\xi^{+}\right)\right) \\
& +c\left(\xi^{+}\right)+\bar{c}\left(\xi^{-}\right) .
\end{aligned}
$$

from the knowledge of the general solution of the equations of motion associated with a WZW action, i.e ${ }^{4}$.

$$
\begin{aligned}
g\left(\xi^{+}, \xi^{-}\right) & =g_{R}^{T}\left(\xi^{-}\right) g_{L}\left(\xi^{+}\right) \\
g_{R}^{T}\left(\xi^{-}\right) & =\left(\begin{array}{cc}
e^{\bar{c}} & e^{\bar{c}} \bar{b} \\
e^{\bar{c}} \bar{a} & e^{\bar{c}} \bar{a} \bar{b}+e^{-\bar{c}}
\end{array}\right) \\
g_{L}\left(\xi^{+}\right) & =\left(\begin{array}{cc}
e^{c} & e^{c} a \\
e^{c} b & e^{c} a b+e^{-c}
\end{array}\right)
\end{aligned}
$$

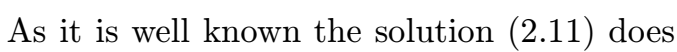
not fix completely $g_{R}, g_{L}(12 . \overline{1})$ which are determined up to a redefinition

$$
g_{L} \rightarrow g_{0} g_{L} \quad g_{R} \rightarrow g_{0}^{-T} g_{R}
$$

we can (partially) fix this invariance by choosing a canonical form for the monodromies. This invariance is also connected to the possibility of using different charts: our parametrization is not global and therefore the group has to be covered with charts where one patch is parametrized as in $\left(\underline{2} . \overline{1}{ }^{2}\right)$ and the others can be chosen to be

$$
\begin{aligned}
& g_{(1) L}\left(\xi^{+}\right)=\left(\begin{array}{cc}
e^{c_{(1)}} b_{(1)} & e^{c_{(1)}} a_{(1)} b_{(1)}+e^{-c_{(1)}} \\
-e^{c_{(1)}} & -e^{c_{(1)}} a_{(1)}
\end{array}\right) \\
& g_{(3) L}\left(\xi^{+}\right)=-\left(\begin{array}{cc}
e^{c_{(3)}} b_{(3)} & e^{c_{(3)}} a_{(3)} b_{(3)}+e^{-c_{(3)}} \\
-e^{c_{(3)}} & -e^{c_{(3)}} a_{(3)}
\end{array}\right) \\
& g_{(2) L}\left(\xi^{+}\right)=-\left(\begin{array}{cc}
e^{c_{(2)}} & e^{c_{(2)}} a_{(2)} \\
e^{c_{(2)}} b_{(2)} & e^{c_{(2)}} a_{(2)} b_{(2)}+e^{-c_{(2)}}
\end{array}\right)
\end{aligned}
$$

with transition function given by $\Omega=\left(\begin{array}{c}1 \\ -1\end{array}\right)$ (i.e. $g_{(i+1) L}=\Omega g_{(i) L}$ with $\left.i \bmod 4\right)$ for $g_{L}$ and

\footnotetext{
${ }^{4}$ The reason why we choose such a parametrization is because we want the canonical momenta associated with $x^{ \pm}$be symmetric in the exchange of the barred and unbarred quantities.
}

similarly for $g_{R}$. If we do not want to use charts we have to use singular functions as it happens with the Dirac monopole.

\subsection{Canonical formalism.}

Since we want to to discuss canonical transformations from interacting fields to Wakimoto ones we need to set up the canonical formalism. This is easily done and we find the momenta

$\mathcal{P}=\frac{|k|}{2 \pi} \dot{\rho} \quad \mathcal{P}_{+}=\frac{|k|}{2 \pi} e^{2 \rho} \partial_{+} x^{-} \quad \mathcal{P}_{-}=\frac{|k|}{2 \pi} e^{2 \rho} \partial_{-} x^{+}$ along with the classical hamiltonian

$\mathcal{H}=\frac{\pi}{|k|} \mathcal{P}^{2}+\frac{|k|}{4 \pi} \rho^{\prime 2}+\frac{4 \pi}{|k|} e^{-2 \rho} \mathcal{P}_{+} \mathcal{P}_{-}-\mathcal{P}_{-} x^{\prime-}+\mathcal{P}_{+} x^{\prime+}$

Moreover we can write the non vanishing Poisson brackets as

$$
\begin{aligned}
\left\{x^{+}(\sigma), \mathcal{P}_{+}\left(\sigma^{\prime}\right)\right\} & =\left\{x^{-}(\sigma), \mathcal{P}_{-}\left(\sigma^{\prime}\right)\right\}=\delta\left(\sigma-\sigma^{\prime}\right) \\
\left\{\rho(\sigma), \mathcal{P}\left(\sigma^{\prime}\right)\right\} & =\delta\left(\sigma-\sigma^{\prime}\right)
\end{aligned}
$$

Obviously this expressions are not very useful because we cannot use them to deduce the commutation relations between the "oscillators" $a, b, c$ and $\bar{a}, \bar{b}, \bar{c}$ due to the highly non linear way they enter the expressions for $x^{ \pm}, \rho$, explicitly

$$
\mathcal{P}_{+}=\frac{|k|}{2 \pi} e^{2 c} \partial_{+} b \quad \mathcal{P}_{-}=\frac{|k|}{2 \pi} e^{2 \bar{c}} \partial_{-} \bar{b}
$$

\subsection{The KM algebra and the energy-momentum tensor.}

From the standard classical expression for the left/right KM currents $J=|k| g^{-1} \partial_{+} g\left(\bar{J}=|k| \partial_{-} g g^{-1}\right)$ we can compute the classical KM currents which read

$$
\begin{aligned}
J^{-} & =|k| e^{2 \rho} \partial_{+} x^{-} \\
J^{3} & =|k|\left(\partial_{+} \rho-x^{+} e^{2 \rho} \partial_{+} x^{-}\right) \\
J^{+} & =|k|\left(\partial_{+} x^{+}+2 x^{+} \partial_{+} \rho-x^{+2} e^{2 \rho} \partial_{+} x^{-}\right)
\end{aligned}
$$

and

$$
\begin{aligned}
& \bar{J}^{-}=|k|\left(\partial_{-} x^{-}+2 x^{-} \partial_{-} \rho-x^{-2} e^{2 \rho} \partial_{-} x^{+}\right) \\
& \bar{J}^{3}=|k|\left(\partial_{-} \rho-x^{-} e^{2 \rho} \partial_{-} x^{+}\right) \\
& \bar{J}^{+}=|k| e^{2 \rho} \partial_{-} x^{+}
\end{aligned}
$$


The previous currents can be rewritten in the canonical formalism in the following way

$$
\begin{aligned}
J^{-}= & 2 \pi \mathcal{P}_{+} \\
J^{3}= & \frac{|k|}{2}\left(\frac{2 \pi}{|k|} \mathcal{P}+\rho^{\prime}\right)-2 \pi x^{+} \mathcal{P}_{+} \\
J^{+}= & |k| x^{\prime+}+|k| x^{+}\left(\frac{2 \pi}{|k|} \mathcal{P}+\rho^{\prime}\right) \\
& -2 \pi x^{+2} \mathcal{P}_{+}+2 \pi e^{-2 \rho} \mathcal{P}_{-} \\
\bar{J}^{-}= & -|k| x^{\prime-}+|k| x^{-}\left(\frac{2 \pi}{|k|} \mathcal{P}_{-} \rho^{\prime}\right) \\
& -2 \pi x^{-2} \mathcal{P}_{-}+2 \pi e^{-2 \rho} \mathcal{P}_{+} \\
\bar{J}^{3}= & \frac{|k|}{2}\left(\frac{2 \pi}{|k|} \mathcal{P}_{-} \rho^{\prime}\right)-2 \pi x^{-} \mathcal{P}_{-} \\
\bar{J}^{+}= & 2 \pi \mathcal{P}_{-}
\end{aligned}
$$

while the momentum-energy tensor reads

$$
\begin{aligned}
T_{++}= & \frac{|k|}{4}\left(\frac{2 \pi}{|k|} \mathcal{P}+\rho^{\prime}\right)^{2}+\frac{4 \pi^{2}}{|k|} e^{-2 \rho} \mathcal{P}_{+} \mathcal{P}_{-} \\
& +2 \pi \mathcal{P}_{+} x^{\prime+} \\
T_{--}= & \frac{|k|}{4}\left(\frac{2 \pi}{|k|} \mathcal{P}-\rho^{\prime}\right)^{2}+\frac{4 \pi^{2}}{|k|} e^{-2 \rho} \mathcal{P}_{+} \mathcal{P}_{-} \\
& -2 \pi \mathcal{P}_{-} x^{\prime-}
\end{aligned}
$$

It is then an easy matter to verify that they satisfy the following classical Virasoro (with vanishing central charge)

$$
\begin{array}{r}
\left\{T_{++}(\sigma), T_{++}\left(\sigma^{\prime}\right)\right\}= \\
2 \pi\left[T_{++}(\sigma)+T_{++}\left(\sigma^{\prime}\right)\right] \partial_{\sigma} \delta\left(\sigma-\sigma^{\prime}\right) \\
-2 \pi \frac{c}{12} \partial_{\sigma}^{3} \delta\left(\sigma-\sigma^{\prime}\right) \\
\left\{T_{--}(\sigma), T_{--}\left(\sigma^{\prime}\right)\right\}= \\
-2 \pi\left[T_{--}(\sigma)+T_{--}\left(\sigma^{\prime}\right)\right] \partial_{\sigma} \delta\left(\sigma-\sigma^{\prime}\right) \\
+2 \pi \frac{c}{12} \partial_{\sigma}^{3} \delta\left(\sigma-\sigma^{\prime}\right)
\end{array}
$$

with $c=0$ and Kac-Moody algebra (of level $|k|$ )

$$
\begin{aligned}
\left\{J^{a}(\sigma), J^{b}\left(\sigma^{\prime}\right)\right\}= & 2 \pi \epsilon_{\ldots c}^{a b} J^{c} \delta\left(\sigma-\sigma^{\prime}\right) \\
& +\pi|k| \eta^{a b} \delta^{\prime}\left(\sigma-\sigma^{\prime}\right)(2.27) \\
\left\{\bar{J}^{a}(\sigma), \bar{J}^{b}\left(\sigma^{\prime}\right)\right\}= & -2 \pi \epsilon_{\ldots c}^{a b} \bar{J}^{c} \delta\left(\sigma-\sigma^{\prime}\right) \\
& -\pi|k| \eta^{a b} \delta^{\prime}\left(\sigma-\sigma^{\prime}\right)(2.28)
\end{aligned}
$$

It is not difficult to check the classical Sugawara construction, i.e. $T=\frac{1}{|k|} \eta_{a b} J^{a} J^{b}$.

\subsection{The classical canonical transformation to the Wakimoto fields.}

We are now ready to discuss the classical Wakimoto canonical fields. In order to get a hint on how they are related to our starting canonical variables we evaluate $T$ on the general solution of the equations of motion and we get

$$
T_{++}=|k|\left(\partial_{+} c\right)^{2}+|k| e^{2 c} \partial_{+} b \partial_{+} a
$$

Remembering the value of $\mathcal{P}+$ given in eq. (12.15 the previous expression suggests that $c$ has something to do with a "free" field while $a$ could be proportional to the field canonically conjugate to $\mathcal{P}_{+}$. Starting from this observation it is not difficult to show that

$$
\begin{aligned}
& F=\sqrt{2|k|}(c+\bar{c}) \\
& \beta=2 \pi i \mathcal{P}_{+}, \gamma=a \\
& \bar{\beta}=2 \pi i \mathcal{P}_{-}, \bar{\gamma}=\bar{a}
\end{aligned}
$$

which satisfy the following canonical Poisson brackets

$$
\begin{aligned}
\left\{F(\sigma), \dot{F}\left(\sigma^{\prime}\right)\right\} & =4 \pi \delta\left(\sigma-\sigma^{\prime}\right) \\
\left\{\beta(\sigma), \gamma\left(\sigma^{\prime}\right)\right\} & =-2 \pi i \delta\left(\sigma-\sigma^{\prime}\right) \\
\left\{\bar{\beta}(\sigma), \bar{\gamma}\left(\sigma^{\prime}\right)\right\} & =-2 \pi i \delta\left(\sigma-\sigma^{\prime}\right)
\end{aligned}
$$

reproduce eq.s ( $(2 \overline{2} . \overline{1} \overline{4})$.

We can now use these new canonical variables to express the classical energy momentum tensor

$$
T_{++}=\frac{1}{2}\left(\partial_{+} F\right)^{2}-i \beta \partial_{+} \gamma
$$

and the classical left $\operatorname{sl}(2, R) \mathrm{KM}$ generators

$$
\begin{aligned}
& J^{-}=-i \beta \quad J^{3}=\sqrt{\frac{|k|}{2}} \partial_{+} F+i \beta \gamma \\
& J^{+}=|k| \partial_{+} \gamma+\sqrt{2|k|} \gamma \partial_{+} F+i \beta \gamma^{2}
\end{aligned}
$$

and the classical right $\operatorname{sl}(2, R)$ ones

$$
\begin{aligned}
& \bar{J}^{+}=-i \bar{\beta} \quad \bar{J}^{3}=-\sqrt{\frac{|k|}{2}} \partial_{-} F+i \bar{\beta} \bar{\gamma} \\
& \bar{J}^{-}=|k| \partial_{-} \bar{\gamma}-\sqrt{2|k|} \bar{\gamma} \partial_{-} F+i \bar{\beta} \bar{\gamma}^{2}
\end{aligned}
$$

\section{Monodromy matrices and bound- ary conditions.}

Which are the boundary conditions to be imposed, here we follow the approach of ( $($ [n] ally and naively we would take $g_{R}, g_{L} \in S L(2, R)$ 
but in this case proceeding in this way we would get some very ugly results or miss two different physical sectors as we are going to explain, we take therefore $g_{R}, g_{L} \in S L(2, C)$ with the further condition dictated by reality of $g$

$$
g_{L}^{*}=g_{C C} g_{L} g_{R}^{*}=g_{R} g_{C C}^{-1} g_{C C} \in S L(2, C) .
$$

We can now ask which is the most general boundary conditions we can impose on $g_{L, R}$ compatible with eq.s (2.6) is the periodicity in $g$ which can be achieved by imposing the following boundary conditions

$$
\begin{aligned}
& g_{L}\left(\xi^{+}+2 \pi\right)=g_{P} g_{L}\left(\xi^{+}\right) \\
& g_{R}\left(\xi^{-}-2 \pi\right)=g_{R}\left(\xi^{-}\right) g_{P}^{-1}
\end{aligned}
$$

A less obvious answer which is nevertheless allowed by the boundary conditions is

$$
\begin{aligned}
& g_{L}\left(\xi^{+}+2 \pi\right)=g_{P} g_{L}\left(\xi^{+}\right) g_{L 0} \\
& g_{R}\left(\xi^{-}-2 \pi\right)=g_{R 0} g_{R}\left(\xi^{-}\right) g_{P}^{-T}
\end{aligned}
$$

where $g_{L 0}=\left(\begin{array}{cc}1 & 2 \pi w \\ 0 & 1\end{array}\right)$ and $g_{R 0}=\left(\begin{array}{cc}1 & 2 \pi \bar{w} \\ 0 & 1\end{array}\right)$ as it can be checked from $(2.6+2.7)$. From the explicit form of the Wakimoto fields it turns out that both $w$ and $\bar{w}$ have vanishing Poisson brackets with all the fields: this is not strange as it can appear because the same happens in the flat limit.

Obviously eq. (3.1) has to be compatible with eq. (3.3), i.e. $g_{C C}$ and $g_{P}$ have to satisfy

$$
g_{C C} g_{P}=g_{P}^{*} g_{C C}
$$

As far as the periodicity is concerned there are three different equivalence classes: $g_{P}$ can be either hyperbolic, parabolic or elliptic. Such classes correspond to tachionic, massless and massive string excitations in the flat limit $k \rightarrow \infty$.

\subsubsection{Hyperbolic sector.}

Let us start with the hyperbolic class is the most natural with the coordinates associated with the Gauss decomposition (2.2i). This case can be described, up to conjugation by a constant element, by taking

$$
g_{P}=\left(\begin{array}{cc}
e^{2 \pi p} & \\
& e^{-2 \pi p}
\end{array}\right) \quad p>0
$$

where the constraint $p>0$ is due to the symmetry $g_{L} \rightarrow \Omega g_{L}$ with $\Omega=\left(\begin{array}{c}1 \\ -1\end{array}\right)$. This form of the periodicity matrix does not fix completely the invariance $\left(\overline{2} . \overline{1} 3_{1}^{1}\right)$ which can, for example, be generically fixed by the further constraint $\bar{b}(0)=b(0)$; analogous considerations apply for the other sectors. This $g_{P}$ is compatible with $g_{C C}=1$.

We can write the explicit expansions of the functions entering the general solution with the given boundary conditions as

$$
\begin{array}{r}
a=w \xi^{+}+a_{\text {periodic }}, b=e^{2 p \xi^{+}} b_{\text {periodic }}, c=p \xi^{+}+c_{\text {periodic }} \Rightarrow \\
F=p \tau+F_{\text {periodic }}, \beta=\beta_{\text {periodic }}, \gamma=w \xi^{+}+\gamma_{\text {periodic }}
\end{array}
$$

similarly for the barred quantities with $\bar{p}=p$ but with $\bar{w}$ independent of $w$. The presence of these two constants $w$ and $\bar{w}$ is allowed by the equality of the variations of $x^{ \pm}$at $\sigma=0,2 \pi$ in particular setting $w=-\bar{w}=R n(n \in Z)$ is equivalent to compactify the $x^{1}$ with radius $R$, i.e. to choose an extremal $\mathrm{BH}$ as background ([1 $\left.\mathrm{i}_{2}^{\prime} \mathrm{O}_{1}^{\prime}\right)$. It is important to notice that both $w$ and $\bar{w}$ have vanishing Poisson brackets with everything as it can be verified from the free field representation. Analogous considerations apply to the other sectors.

It is interesting to notice that this is the only

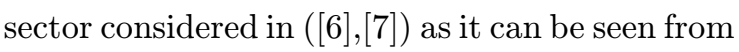
the $F$ expansion.

\subsubsection{Elliptic sector.}

Let us now consider the elliptic case which describes massive excitations in the flat limit where

$g_{P}=\left(\begin{array}{ll}\cos 2 \pi p & \sin 2 \pi p \\ \sin 2 \pi p & \cos 2 \pi p\end{array}\right) \approx\left(\begin{array}{cc}e^{i 2 \pi p} & \\ & e^{-i 2 \pi p}\end{array}\right) 0<p<\frac{1}{2}$

where the first expression is the natural one when restricting the attention to real $g_{L, R}$, i.e. when $g_{C C}=1$ while the second one is the most natural when considering complex $g_{L, R}$ with $g_{C C}=$ $\pm\left(\begin{array}{c}i \\ i\end{array}\right)$

If we insist to use the real fields we get strange and ugly boundary conditions such as

$c\left(\xi^{+}+2 \pi\right)=c\left(\xi^{+}\right)+\log \left(\cos 2 \pi p+b\left(\xi^{+}\right) \sin 2 \pi p\right)$

which propagate to strange, non free boundary conditions for the Wakimoto fields while using 
complex fields we get nice and free boundary conditions since the complex fields have anal-

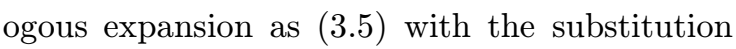
$p \rightarrow i(p+k)$ (where $k \in Z$ ) but it obliges to impose the constraints

$$
a^{*}=a+\frac{1}{b e^{2 c}}, \quad b^{*}=\frac{1}{b}, \quad c^{*}=c+\log ( \pm i b)
$$

They can be imposed in a better way by requiring the reality of the KM currents.

Notice that if we restrict the momentum $p$ to the first Block wave the constraint $0<p<\frac{1}{2}$ implies $0<j=p_{F}<\frac{k}{2}$ which is equivalent to the unitary spectrum truncation at the quantum level.

\subsubsection{Parabolic sector.}

Let us now consider the parabolic case where

$$
g_{P}=\left(\begin{array}{cc}
1 & 0 \\
2 \pi p & 1
\end{array}\right) \quad p \in R
$$

and $g_{C C}=1$, then the fields in the 0th patch can be expanded as

\section{Conclusions.}

We have shown that a free fields approach to string propagating on $A d S_{3}$ requires a lot of attention and that we must work with different charts, as done in (陑) for the Liouville theory, if we want to treat the parabolic sector correctly. As byproducts of this analysis we have shown that the spectrum proposed by ( rally truncated to the hyperbolic sector and that it is possible to describe a string propagating on an extremal $\mathrm{BH}$ background without much effort.

\section{References}

[1] J. Balog, L. O'Raifeartaigh . Forgacs and A. Wips, Consistency of string propagation on curved spacetimes. An $S U(1,1)$ based counterexample, 'Nucl. Phys. B 325 (1989) 225'

[2] P.M.S. Petropoulos, Comments on $S U(1,1)$ string theory, 'Phys. Lett. B_236 (1990) 151',

[3] N. Mohammedi, On the unitarity of string propagation on $S U(1,1)$, Int. J. Mod. Phys. A

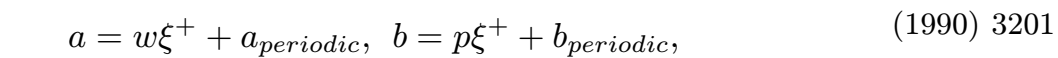

$$
c=c_{\text {periodic }} \Rightarrow F=F_{\text {periodic }}
$$$$
\beta=\beta_{\text {periodic }}, \gamma=\gamma_{\text {periodic }}
$$

[4] S. Hwang, No ghost theorem for $S U(1,1)$ string theories, 'Nucl. Phys. B $\mathbf{3 5 4}(1991) 100$

M. Henningson and S. Hwang, The unitarity of $S U(1,1)$ fermionic strings, 'Phys. Lett. $\mathbf{B} \mathbf{2} 58$ $(1991) 341$

M. Henningson, S. Hwang and P. Roberts, Modular invariance of $S U(1,1)$ string, 'Phys. Lett. $\mathbf{2 6 7}(1991) 350$

[5] J.M. Evans, M.R. Gaberdiel and M.J. Perry, The No-ghost Theorem for $A d S_{3}$ and the Stringy Exclusion Principle, "hep-th/9806024"

[6] I. Bars, Ghost-Free Spectrum of a Quantum String in $S L(2, R)$ Curved Spacetime, 'Phys.। Rev. D3 (1996) 3308; inep-th/9503205

There is apparently not an easy way to impose

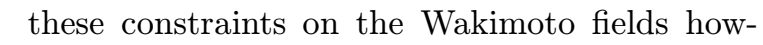
ever it is necessary to use patches in this sector.

A comment is now necessary in order to explain why it is necessary to work with two patches in order the canonical transformation work fine, exactly as it happens for the Liouville case. . If $p_{F} \neq 0$ we can express $b$ using the new canonical variables $F$ and $\beta$ but if we try to solve for $b$ when $p_{F}=0$ then we cannot recover $b_{0}$ (the - constant mode of $b_{\text {periodic }}^{---}$from the expression for $\bar{\beta}$. This can clearly be avoided if we use two patches.
Solution of the $S \bar{L}(2, R)$ string in curved spacetime, hep-th/9511187

[7] Y. Satoh, Ghost-free and modular invariant spectra of a string in $S L(2, R)$ and three dimensional black hole geometry, 'Nucl. Phys. B $\mathbf{5 1 3}$ (1998) 213; ', 'hep-th/9705208'

[8] J.L. Gervais and A. Neveu, The Dual String Spectrum in Polyakov's Quantization (I), 'Nucl.' Phys. B $199(1982) 59$

The Dual String Spectrum in Polyakov's Quantization (II), 'Nucl. Phys. B 209 (1982) 125! 
[9] P.M.S. Petropoulos, String Theory_on $A d S_{3}$ : some open Questions, hep-th/9908189',

[10] J. Maldacena and A. Strominger, $A d S_{3}$ black

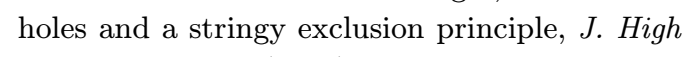
- _ _ Energy Phys. 12 (1998)_005

[11] J. Maldacena and H. Ooguri, Strings in $A d S_{3}$ and $S L(2, R)$ WZW model: I, hep-th/0001053',

[12] A.L. Larsen and N. Sanchez. Quantum coherent string states in $A d S_{3}$ and $S L(2, R)$ WZWN model, hep-th/0001180,

[13] A. Kato and Y. Satoh, Modular invariance of string theory on $A d S_{3}$, hep-th/0001063'

[14] I. Tsutsui and L. Feher, Global aspects of the WZWN reduction to Toda theories, Prog.Theor.Phys.Suppl. 118 (1995) 173, hep-th/9408065 